\title{
Suppression of Dendrite Formation via Pulse Charging in Rechargeable Lithium Metal Batteries
}

\author{
Matthew Z. Mayers, Jakub W. Kaminski, and Thomas F. Miller, III* \\ Division of Chemistry and Chemical Engineering, California Institute of Technology, Pasadena, California 91125, United States
}

\begin{abstract}
We introduce a coarse-grained simulation model for the reductive deposition of lithium cations in secondary lithium metal batteries. The model accounts for the heterogeneous and nonequilibrium nature of the electrodeposition dynamics, and it enables simulation of the long timescales and lengthscales associated with metal dendrite formation. We investigate the effects of applied overpotential and material properties on earlystage dendrite formation, as well as the molecular mechanisms that

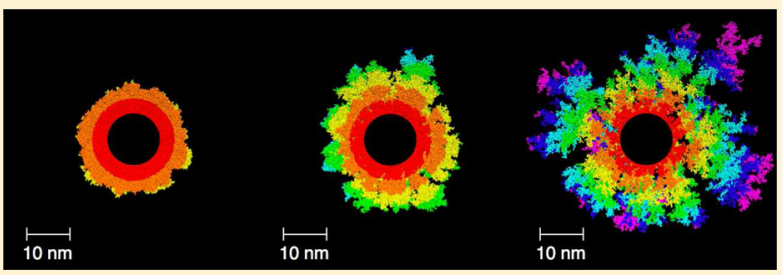
govern this process. The model confirms that dendrite formation propensity increases with the applied electrode overpotential, and it demonstrates that application of the electrode overpotential in time-dependent pulses leads to dramatic suppression of dendrite formation while reducing the accumulated electrode on-time by as much as $96 \%$. Moreover, the model predicts that time dependence of the applied electrode overpotential can lead to positive, negative, or zero correlation between cation diffusivity in the solid-electrolyte interphase (SEI) and dendrite formation propensity. Analysis of the simulation trajectories reveals that dendrite formation emerges from a competition between the timescales for cation diffusion and reduction at the anode/SEI interface, with lower applied overpotentials and shorter electrode pulse durations shifting this competition in favor of lower dendrite formation propensity. This work provides a molecular basis for understanding and designing pulsing waveforms that mitigate dendrite formation while minimally affecting battery charging times.
\end{abstract}

\section{INTRODUCTION}

As the lightest and most electropositive metal, lithium is an appealing battery anode material, and lithium metal batteries find use in many portable electronic devices. ${ }^{1}$ However, the development of secondary lithium metal batteries is hindered by the propensity of lithium to deposit in dendritic structures during battery recharging; the formation and growth of these structures is a source of battery failure. ${ }^{2}$ It is well-known that dendrite formation is correlated with factors including high current densities, ${ }^{3-5}$ electrolyte salts, ${ }^{6}$ and electrolyte solvent composition. ${ }^{6}$ However, the detailed molecular mechanisms associated with dendrite nucleation and growth remain poorly understood, and the mitigation of dendrite formation remains an important experimental and industrial challenge.

Experimental studies have demonstrated that pulse charging alters electrodeposition morphologies in a variety of metals, suggesting new methods for dendrite suppression. ${ }^{7}$ Pulse charging, in which electrode overpotentials are applied periodically in time, has been reported to increase the cycle life of alkaline ${ }^{8}$ and lithium ion ${ }^{9}$ batteries. The use of timedependent applied overpotentials can lead to target compositions, structures, and porosities of electrode deposition structures, while reducing energy waste due to internal resistance and reducing the need for electrolyte additives. ${ }^{10,11}$ Various trends that associate deposition morphology with duty cycle and pulsing frequency have been empirically identified, ${ }^{8}$ but the underlying molecular processes that determine the optimal pulse charging waveform remain unclear.

The development of molecular simulation methods to understand and explore lithium dendrite formation provides a powerful approach to addressing these questions. A variety of lattice-based models have been employed to study shortlengthscale dendrite formation. ${ }^{12-16}$ For example, coarsegrained (CG) lattice models in three dimensions have been shown to reproduce analytical expressions for concentration profiles and cell current, ${ }^{12}$ whereas two- and three-dimensional CG models have examined the morphology and growth rate of dendrites as a function of applied overpotential and the distribution of nucleation sites along the anode. ${ }^{13,14}$ Furthermore, electronic network models have been developed to study deposition dynamics and heat generation using continuum representations for the system. ${ }^{9}$ However, particlebased simulations of pulse charging in an electrochemical cell have not been previously reported.

The long timescales and large lengthscales associated with lithium dendrite formation present significant challenges from the perspective of molecular simulation. The dynamics of electrodeposition includes processes that range from ion diffusion to electron-transfer reactions to surface reorganization; these processes span from picosecond to minute timescales and involve up to millions of atoms, exceeding the scope of atomistic simulation methods. In the current study, we develop a particle-based CG model of a lithium metal anode that enables the efficient characterization of electrodeposition and dendrite formation. The model allows for the direct simulation of electrochemical deposition, as well as examination

Received: September 19, 2012

Revised: November 13, 2012

Published: November 19, 2012 
of the effects of different pulse-charging waveforms on deposition morphology. We investigate the effects of pulse duration and duty cycle of the waveform on deposition morphology, and we determine the mechanistic origin of these trends. This work provides new insights into the competition of molecular processes associated with anodal electrodeposition during battery recharging, and it provides a simulation-based framework for developing improved potential waveforms for pulse charging.

\section{METHODOLOGY}

Here, we introduce the CG model for simulating the nonequilibrium electrodeposition of cations onto a nanoparticle surface. Key components of the model include off-lattice dynamics of ions in the solid-electrolyte interphase (SEI), stochastic ion-reduction and deposition events associated with electron-transfer reactions at the electrode/SEI interface, and a particle bath that reproduces the bulk cation concentration at large distances from the electrode. The CG model allows for the efficient simulation of reactive, second-timescale molecular dynamics (MD) trajectories associated with early stage metal dendrite nucleation and growth, and it enables the quantitative investigation of the dependence of dendrite formation propensity on experimental conditions and material parameters, such as ion diffusivity, the electrolyte dielectric response, and the electrode overpotential waveform, frequency, and magnitude. Details of the CG model are now described.

The System. We consider cation electrodeposition onto a hemispherical electrode that is attached to an inert, planar surface (Figure 1A). All simulations are performed using an electrode of radius $R=6.00 \mathrm{~nm}$, and the total simulation cell is truncated at a distance of $S=44.4 \mathrm{~nm}$ from the electrode origin. Metal cations in the SEI (Figure 1A, blue) and metal atoms that are formed via reductive deposition of the cations (Figure 1A, black) are explicitly represented. Counterion species are only implicitly included via electrostatic screening.

The SEI is a passivating layer ranging in thickness from 5 to $50 \mathrm{~nm}$ that forms spontaneously in lithium ion and lithium metal batteries as a result of chemical reactions between the anode and the electrolyte solution. ${ }^{17}$ It exhibits properties of a solid electrolyte and has been shown to be a critical factor in determining the morphology of electrodeposition, ${ }^{18,19}$ although it remains a poorly understood feature of battery interfaces. ${ }^{20}$ For simplicity in the current simulations, the SEI extends to all regions of the simulation cell beyond the surface of the anode.

The model system employed in this study corresponds to recent experiments involving nanoparticle-catalyzed singlemolecule electrochemistry, ${ }^{21,22}$ as well as studies that employ nanoparticle anodes for energy-grid applications. ${ }^{23}$ Aside from relatively minor effects associated with the nanoparticle anode curvature, ${ }^{24}$ we expect that the trends and molecular mechanisms elucidated here will also apply to electrodeposition at a planar electrode. We note that the model may easily be extended to study other strategies for suppressing lithium metal dendrites, including cells that incorporate block copolymer electrolytes, such as those investigated by Balsara and coworkers. $^{25}$

Cation Interactions and Diffusive Dynamics. Prior to reductive plating, the metal cations evolve according to overdamped stochastic dynamics, which are simulated using the forward Euler integration scheme, ${ }^{26}$

$$
\mathbf{r}_{i}(t+\Delta t)=\mathbf{r}_{i}(t)+(2 D \Delta t)^{1 / 2} \mathbf{g}_{i}
$$
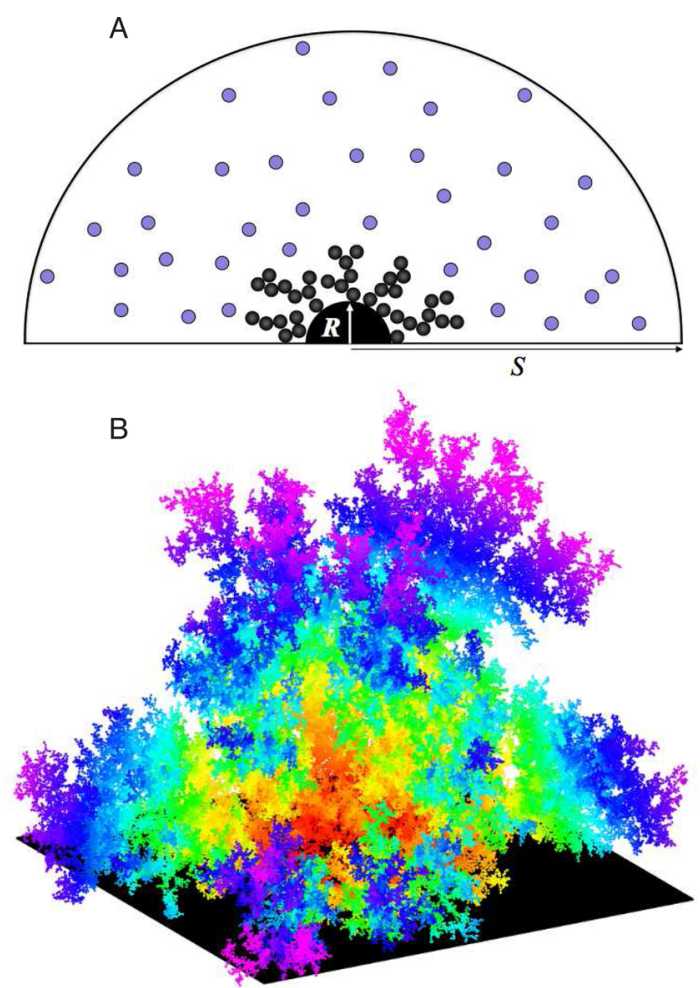

Figure 1. CG model for simulating the electrodeposition of cations onto a nanoparticle anode surface. (A) Schematic cross-section of the hemispherical simulation cell. Blue particles represent metal cations diffusing in the SEI, and black particles represent metal atoms that have deposited onto the metal nanoparticle of radius $R$, shown as a black hemisphere. (B) Snapshot of the simulation cell at the end of a deposition trajectory, which employs continuous charging with reaction probability $p=1$. The deposited metal atoms are colored according to their distance from the nanoparticle anode, and the inert planar surface is shown in black.

where $\mathbf{r}_{i}(t)$ is the three-dimensional (3D) position vector for cation particle $i$ at time $t, \mathbf{g}_{i}$ is a $3 \mathrm{D}$ vector of normally distributed random variables, $D$ is the isotropic cation diffusion coefficient, and $\Delta t$ is the MD time step. Employed values for these parameters are reported in Table 1 . The value for the

\section{Table 1. Simulation Parameters}

\begin{tabular}{ll}
\multicolumn{1}{c}{ parameter } & value ranges \\
particle radius, $a(\mathrm{~nm})$ & 0.119 \\
diffusion coefficient, $D\left(\mathrm{~m}^{2} \mathrm{~s}^{-1}\right)$ & $1.40 \times 10^{-14}$ \\
concentration, $C(\mathrm{M})$ & 0.174 \\
anode radius, $R(\mathrm{~nm})$ & 6.00 \\
outer cell radius, $S(\mathrm{~nm})$ & 44.4 \\
time step, $\Delta t(\mu \mathrm{s})$ & 0.253 \\
reaction probability, $p$ & $10^{-3}$ to 1 \\
particle bath timescale, $\tau(\mathrm{ms})$ & 0.253 \\
anode on-time, $t_{\mathrm{on}}(\mu \mathrm{s})$ & $2.53-25300$ \\
anode off-time, $t_{\mathrm{off}}$ & $t_{\mathrm{on}}-100 t_{\mathrm{on}}$ \\
\hline
\end{tabular}

diffusion coefficient employed in the simulations corresponds to the measured current flow of lithium cations in propylenecarbonate-based solutions, ${ }^{27}$ which model the complex SEI environment. We note that common SEI components can vary substantially in ion diffusivity, ${ }^{28}$ the effect of which is explored 
in the Double Layers, Diffusion Timescales, and Pulse Charging section of the Results.

Interactions among the metal cations in the SEI are screened by implicit counterions; at the bulk ion concentrations considered here, the average distance between the explicit cations $(\sim 2 \mathrm{~nm})$ is much larger than that of the isotropic Debye screening length $(\sim 0.6 \mathrm{~nm}$ for the conditions employed in these simulations). The metal cations exhibit hard-wall interactions with the planar surface, with all reduced metal atoms, and with the two hemispherical boundaries of the simulation cell at distances of $R$ and $S$ from the electrode origin. In practice, these hard-wall interactions are implemented by rejecting $\mathrm{MD}$ position updates for a given cation that introduce an overlap between the finite cation volume and the specified regions; the volume associated with each cation or deposited metal atom is enclosed within a radius $a=0.119 \mathrm{~nm}$ of the corresponding particle position.

As in other models for reductive electrodeposition, ${ }^{12-16}$ attractive interactions between metal cations and either the electrode or the reduced metal atoms on the electrode are neglected. The neglect of these forces (as well as the associated ion-migration flux) is justified by charge screening on short lengthscales. ${ }^{24,29}$

Cation Reduction and Plating. Electrodeposition of cations at the metal electrode occurs as a result of electrontransfer events across the electrode/SEI interface. To model this process, a stochastic cation reduction event is attempted when an $\mathrm{MD}$ move generated by eq 1 leads to overlap between the volume of a metal cation and that of the electrode or a reduced metal atom. The probability for accepting the reduction event is denoted $p$. Position updates and deposition attempts are applied sequentially to particles in these simulations; both steps are performed for a given particle before the subsequent particle position is updated.

If the reduction attempt fails, then the position update from eq 1 is rejected, and the cation is returned to its prior position. If the attempt succeeds, the cation is irreversibly switched to a reduced metal atom and is deposited onto the electrode or other metal atoms. Upon deposition, the position of the new metal atom is adjusted using the following protocol to account for the finite volume of the metal atoms. ${ }^{13}$ In a first step, if the volume of the new metal atom overlaps with that of the hemispherical electrode, then the position of the atom is radially shifted outward until this overlap is eliminated. In a second step, if the volume of the new metal atom overlaps with that of at least one other metal atom, one of the existing metal atoms is randomly chosen, and the position of the new metal atom is shifted along the vector $\mathbf{r}_{\mathrm{n}}-\mathbf{r}_{\mathrm{o}}$ until the overlap is eliminated; here, $\mathbf{r}_{\mathrm{n}}$ and $\mathbf{r}_{\mathrm{o}}$ indicate the respective positions of the new and previously plated metal atoms. This two-step cycle is repeated until the new metal atom overlaps with neither the hemispherical electrode nor any of the previously plated metal atoms. If these overlaps persist for more than 50 cycles, the stochastic reduction event is rejected and the metal cation particle is returned to its original position; in practice, rejection occurs in less than $0.1 \%$ of plating events. Upon plating, the positions of metal atoms remain subsequently unchanged; we thus assume that reorganization (or annealing) timescales for the deposited metal atoms are slow in comparison to the timescales for cation reduction and diffusion. Figure 1B depicts the deposition structure at the end of a typical electrodeposition trajectory.
The reaction probability $p$ incorporates material-dependent properties and experimental conditions via

$$
p=1-\mathrm{e}^{-k_{\mathrm{ET}}(r, \eta) \Delta t}
$$

where $k_{\mathrm{ET}}(r, \eta)$ is the overpotential-dependent rate for electron transfer, ${ }^{24,30}$

$$
k_{\mathrm{ET}}(r, \eta)=\tau_{\mathrm{sol}}^{-1} \kappa_{\mathrm{el}}(r, \eta) \mathrm{e}^{-\Delta G^{\ddagger}(r, \eta) /\left(k_{\mathrm{B}} T\right)}
$$

The prefactor terms $\tau_{\mathrm{sol}}$ and $\kappa_{\mathrm{el}}(r, \eta)$ are related to the timescale for solvent fluctuations and the probability of reactive electron tunneling, respectively. $\Delta G^{\ddagger}(r, \eta)$ is the free energy for the solvent reorganization to the electron-transfer transition state. Related interpretations for stochastic reduction probabilities have been previously described. ${ }^{15,31}$

Nonequilibrium Electrodeposition. A particle bath is employed to replenish cations in the SEI that undergo deposition. At time intervals of $\tau$, new particles are inserted in the hemispherical shell bounded by the hemispheres of radii $T+60 a$ and $S$, where $T$ is the largest distance between the origin and a deposited metal atom. Particles are randomly and uniformly distributed until the cation number density in the particle bath reaches the simulation concentration $C=0.174 \mathrm{M}$, the same cation concentration at which the initial system is equilibrated. This value is typical of cation concentrations in electrochemical cells; ${ }^{32}$ test simulations performed using a 4fold increase in $C$ found no qualitative change in the reported results.

We have confirmed that the chosen distance of separation between the particle bath and the deposition structure (i.e., $60 a$ ) does not affect the simulations; all values for this distance in the range of $0 a$ to $150 a$, where $a$ is the particle radius, are found to yield essentially identical simulation results.

Electrodeposition trajectories are continued until the density of plated particles at the hemispherical surface of radius $R+50 a$ reaches a constant value with respect to time, which requires approximately $1-10 \mathrm{~s}$. We note that the timescales of the simulations are similar to those needed to reach steady-state cation concentration profiles near the anode surface, and the lengthscales of the simulation cell are similar to the approximately $40 \mathrm{~nm}$ lengthscale associated with the steadystate diffusion layer in the cation concentration profile for a spherically symmetric system. ${ }^{24}$ Although the results presented here focus on the early-stage, non-steady-state formation of dendrites, the current model could also be applied to longer length- and timescale processes, such as dendrite propagation, that have been characterized using continuum modeling. ${ }^{33,34}$ However, we note that early stage dendrite formation is of particular interest because the growth of dendrites is difficult to halt once the propagation stage is reached. ${ }^{31}$

Time dependence of the electrode potential is introduced by defining two additional parameters $t_{\text {on }}$ and $t_{\text {off }}$, with which a periodic, square-wave potential is fully specified. During the $t_{\mathrm{on}}$ portion of a period, deposition takes place as described above. During the $t_{\text {off }}$ portion of the waveform, no deposition takes place; reduction attempts are accepted with zero probability.

Individual trajectories typically required $2-72 \mathrm{~h}$ of CPU time on a single $2.66 \mathrm{GHz}$ Intel Xeon E5430 processor.

\section{RESULTS}

The CG model is employed to investigate the relationship between electrode charging conditions and deposition morphology. Electrodeposition trajectories are performed to 
explore effects due to the magnitude of the electrode overpotential, the time dependence of the electrode overpotential, and the cation diffusivity in the SEI medium.

Deposition via Continuous Charging. We begin by considering deposition simulations in which time-independent overpotentials are applied at values corresponding to $p \in$ $\{0.001,0.01,0.03,0.1,0.3,0.5,1\}$. Figure 2 presents snapshots from individual deposition trajectories performed with increasing values of $p$. Each of the snapshots corresponds to the time at which approximately 100000 lithium ions have plated on the nanoparticle electrode. It is clear that the structural features of the deposition structure are strongly

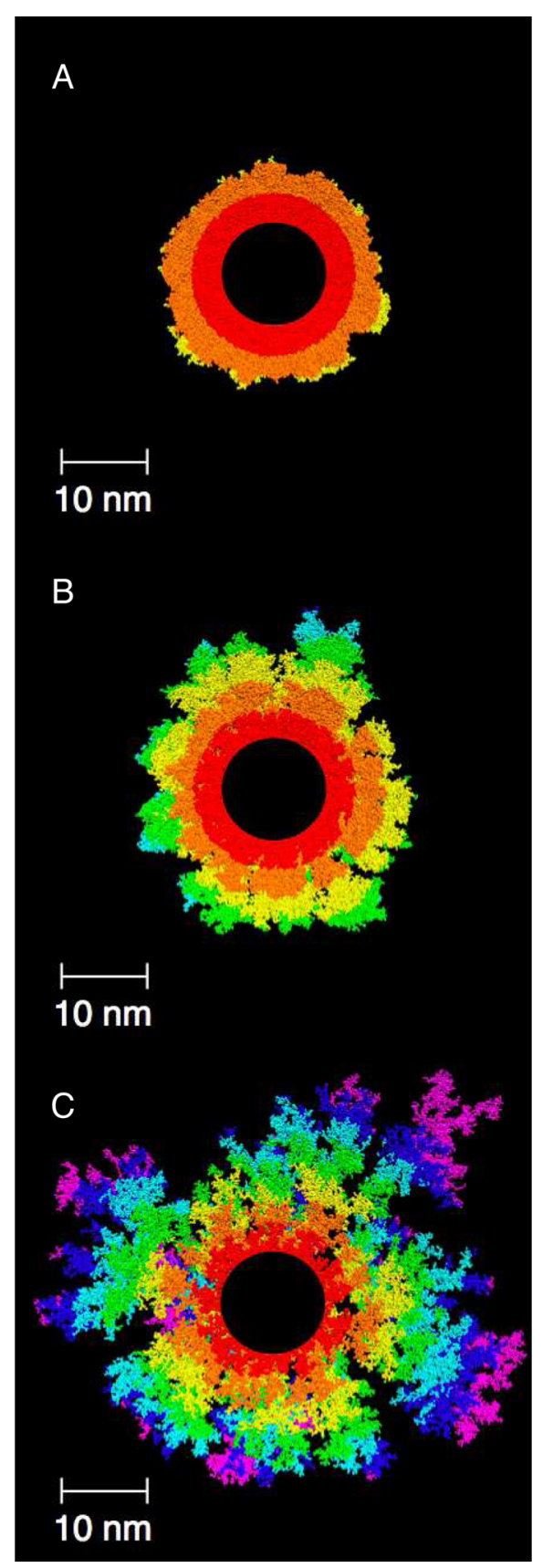

Figure 2. Snapshots of the simulation cell for CG trajectories that employ continuous charging with (A) $p=0.01$, (B) $p=0.1$, and (C) $p$ $=1.0$. The deposition structure is viewed from below the inert planar surface, with deposited metal atoms colored as in Figure 1B. dependent on $p$, with more compact structures corresponding to low overpotentials and with more diffuse structures corresponding to higher overpotentials.

Trajectory-averaged results are shown in Figure 3. Each curve in the figure corresponds to the average of 14 independent
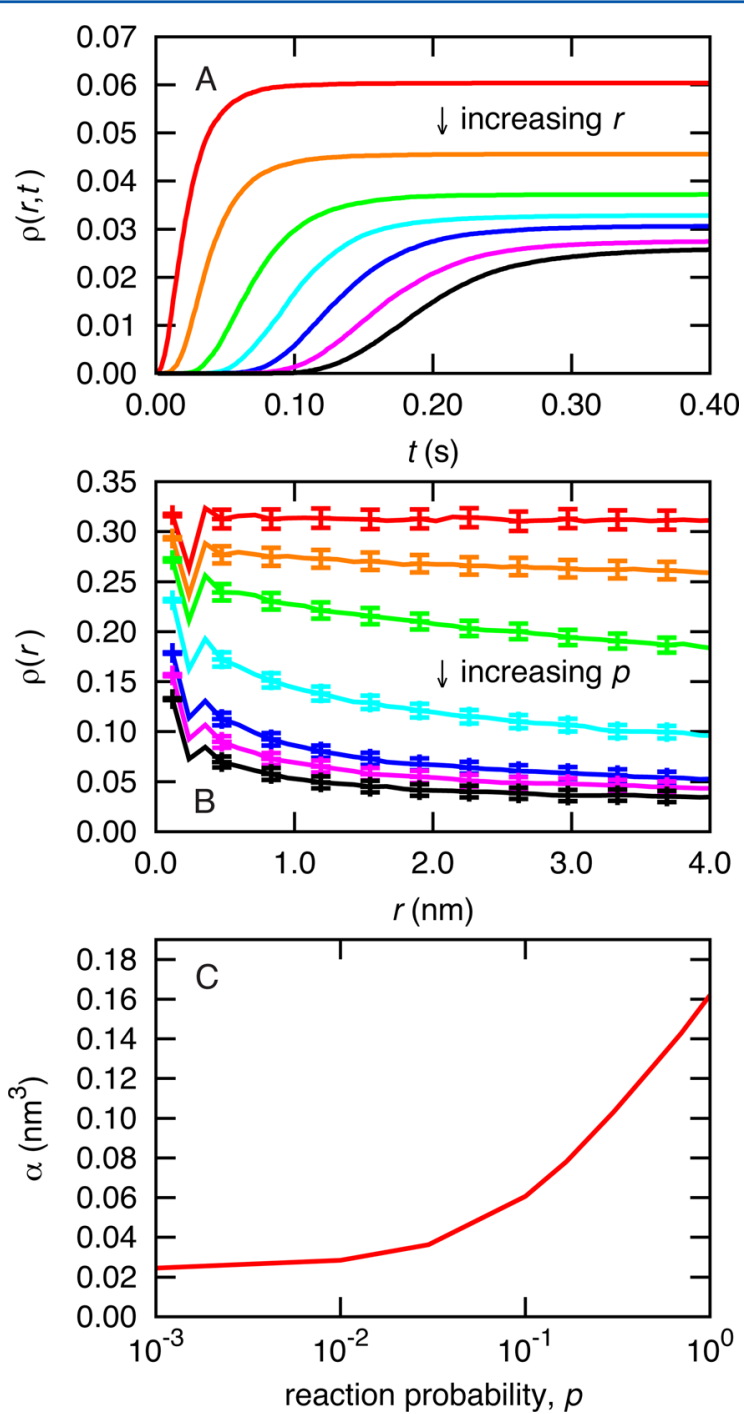

Figure 3. Simulation results obtained with constant applied overpotentials (i.e., continuous charging). (A) The distance- and timedependent deposition density $\rho(r, t)$ is plotted as a function of simulation time at various $r$, with $p=1$ and with $r=0.6 \mathrm{~nm}$ (red), $r=$ $1.2 \mathrm{~nm}$ (orange), $r=2.4 \mathrm{~nm}$ (green), $r=3.6 \mathrm{~nm}$ (light blue), $r=4.8$ $\mathrm{nm}$ (dark blue), $r=6.0 \mathrm{~nm}$ (pink), and $r=7.2 \mathrm{~nm}$ (black). In each case, the error bars are on the order of the thickness of the plotted lines. (B) The long time limit of the deposition density $\rho(r)$ is plotted as a function of $r$. The various curves correspond to results obtained using different reaction probabilities, with $p=0.001$ (red), $p=0.01$ (orange), $p=0.03$ (green), $p=0.1$ (light blue), $p=0.3$ (dark blue), $p$ $=0.5$ (pink), and $p=1$ (black). (C) The dendrite propensity coefficient $\alpha$ is plotted as a function of reaction probability.

deposition trajectories. Figure 3A presents $\rho(r, t)$, the distanceand time-dependent deposition density computed from trajectories with reaction probability $p=1$. This quantity represents the fraction of the surface area of the hemispherical shell of radius $R+r$ that intersects plated particles at time $t$, where $r$ indicates the minimum distance from a given point to the surface of the hemispherical anode. The density is plotted 
as a function of time for the radial shells associated with various $r$.

Figure $3 \mathrm{~A}$ shows that at each distance from the anode surface, the deposition density increases monotonically as a function of time. Plated particles remain fixed in the simulations, so any particle density that has accumulated by time $t$ persists for the remainder of the simulation. At longer times, as the deposition structure expands outward into the SEI, the inner sections of the deposition structure become inaccessible to metal ions in the SEI. The deposition density at a given $r$ remains unchanged at times beyond which cations can no longer sufficiently diffuse into the deposition structure to reach that distance from the anode.

Figure $3 \mathrm{~B}$ presents the long time limit of the deposition density,

$$
\rho(r)=\lim _{t \rightarrow \infty} \rho(r, t)
$$

plotted for a range of values of the reaction probability $p$. For small $p$, the deposition density is relatively insensitive to the distance from the anode, but for larger $p, \rho(r)$ decreases substantially with increasing $r$. Figure $3 \mathrm{C}$ presents the inverse of the average particle number density in the region of the anode surface, obtained using

$$
\alpha^{-1}=V_{R}^{-1} V_{0}^{-1} \int_{R}^{R+30 a} 2 \pi r^{2} \rho(r) \mathrm{d} r
$$

where $V_{R}=\int_{R}^{R+30 a} 2 \pi r^{2} \mathrm{~d} r$ and $V_{0}={ }^{4} /{ }_{3} \pi a^{3}$. The quantity $\alpha$ increases as the deposition structure becomes more loosely packed and dendritic; it is thus termed the dendrite propensity coefficient. Figure 3C shows that greater propensity for dendrite formation is predicted at high reaction probabilities.

Figure 4 illustrates that the mechanistic basis for the observed trend in Figure $3 \mathrm{C}$ is a competition between the timescale for diffusion of cations within the deposition structure and the timescale for reductive plating of the cations onto the deposition structure. Under conditions of low reaction probability, illustrated in Figure 4A, reductive plating occurs

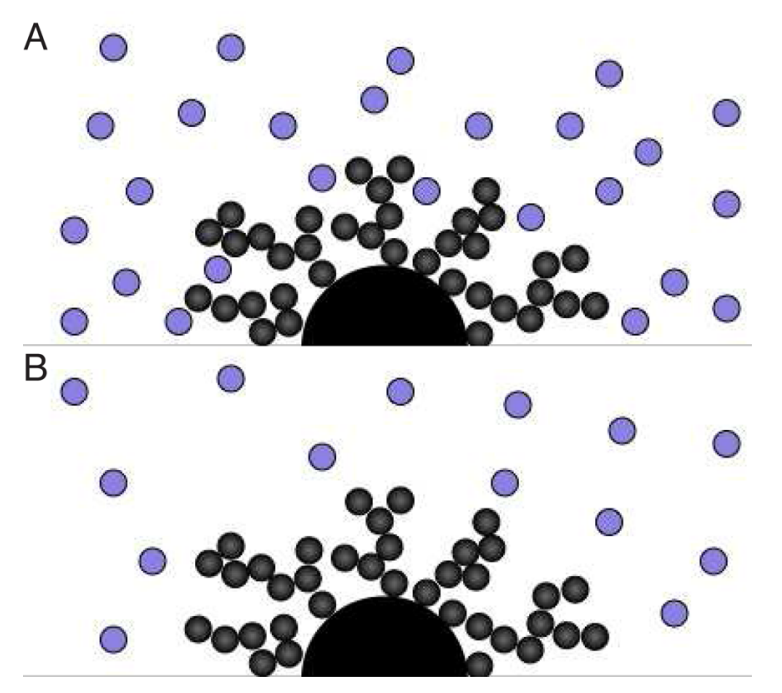

Figure 4. Schematic illustration of the competition between timescales for cation diffusion and reduction. (A) For conditions that favor cation diffusion, cation particles penetrate deeply into the deposition structure before undergoing reduction. (B) For conditions that favor cation reduction, cation particles do not penetrate the deposition structure before undergoing reduction. slowly and the cations diffuse extensively into the deposition structure before undergoing reduction; the result is a dense deposition structure and a low propensity for dendrite formation. Under conditions of high reaction probability, illustrated in Figure 4B, the timescale for reductive plating wins out, and cations have little opportunity to diffuse into the deposition structure before undergoing reductive plating; this leads to a diffuse deposition structure and a high propensity for dendrite formation.

Given the relationship between applied overpotential and the stochastic reaction probability (eqs 2 and 3), the results in Figure $3 \mathrm{C}$ are consistent with experimental observations that dendrite formation decreases with overpotential. ${ }^{3,4}$ For some electrode materials, such as zinc, the use of lower overpotentials to modulate deposition morphology is a practical strategy. ${ }^{35}$ However, for lithium metal batteries, lower overpotentials only slow dendrite formation, rather than preventing it altogether. ${ }^{3}$ The next section explores the use of pulse charging to further reduce dendrite growth.

Deposition via Pulse Charging. We now investigate the effect of time-dependent overpotentials on anodal deposition morphology. Figure 5 presents trajectory-averaged simulation results for a range of pulse-charging parameters. Deposition trajectories are performed with varying pulse durations, $t_{\text {on }} / \Delta t$ $\in\{10,100,1000,10000,100000\}$, and with varying ratios, $\gamma=$ $t_{\text {off }} / t_{\text {on }} \in\{1,5,10,100\}$. Results for each set of pulse-charging parameters are obtained from the average of five independent deposition trajectories. The total length of each deposition trajectory ranges from 1 to $10 \mathrm{~s}$ in time. In all cases, the highoverpotential limit, $p=1$, is employed.

Figure 5A presents the dendrite propensity coefficient, $\alpha$, for the various employed pulse-charging parameters. For comparison, the gray line in Figure 5A indicates the smallest value of $\alpha$ that was reported in Figure $3 \mathrm{C}$ using continuous charging with $p=0.001$. These results show that a decrease in the dendrite propensity coefficient $\alpha$ is seen for both increasing $\gamma$ and decreasing $t_{\text {on }}$. Indeed, in the limit of large $\gamma$ and small $t_{\mathrm{on}} \alpha$ approaches the low-overpotential limit for continuous charging.

Increasing $\gamma$ leads to decreased dendrite propensity in Figure $5 \mathrm{~A}$ because it shifts the competition of timescales in favor of cation diffusion over cation reduction. Longer rest periods between pulses allow for cation diffusion into the deposition structure (as in Figure 4A), such that cation reduction during the pulses leads to high-density deposition. Conversely, increasing $t_{\text {on }}$ shifts the competition of timescales in favor of cation reduction over cation diffusion. As was seen for continuous charging with large overpotentials (Figure 4B), the longer pulses allow less time for cations to diffuse into the deposition structure, such that cation reduction during the pulses leads to low-density deposition. For sufficiently large values of $t_{\text {on }}$, dense plating cannot be attained for any value of $\gamma$ because of the large degree of low-density deposition that occurs within each protracted pulse.

Figure 5B explores the relationship between pulse-charging parameters and the total charging time for the anode, defined here as the amount of time needed for 100000 cation particles to undergo deposition. In general, the total charging time increases as $t_{\text {on }}$ increases and as $\gamma$ increases. However, the results show that total charging times do not increase nearly as quickly as $\gamma$. Figure 5C further illustrates this point by reporting the cumulative amount of time for which deposition is allowed to occur within a deposition trajectory (i.e., total anode ontime) as a function of the pulse-charging parameters $t_{\text {on }}$ and $\gamma$. 

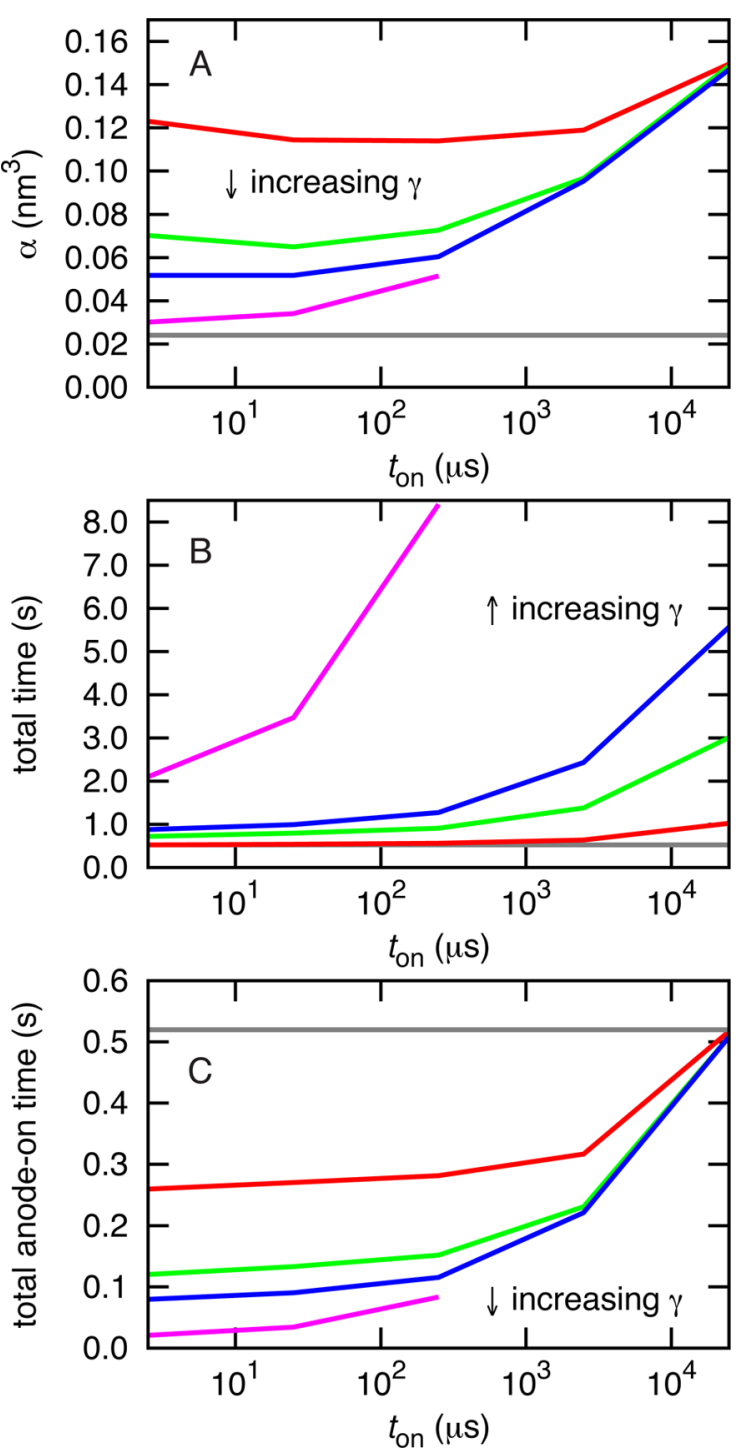

Figure 5. Simulation results obtained with time-dependent applied overpotentials (i.e., pulse charging). (A) Dendrite propensity coefficient $\alpha$ as a function of pulse duration $t_{\mathrm{on}}$. (B) Total simulation time required to deposit 100000 particles, plotted as a function of $t_{\text {on }}$. (C) Total anode on-time required to deposit 100000 particles, plotted as a function of $t_{\mathrm{on}}$. In each panel, the various curves correspond to $\gamma=$ 1 (red), $\gamma=5$ (green), $\gamma=10$ (blue), and $\gamma=100$ (pink), and the error bars are comparable to the thickness of the plotted lines.

In some cases, pulse charging reduces the required anode ontime by as much as $96 \%$ with respect to the time needed for continuous charging.

The observed reduction in anode on-time (Figure 5C) can also be understood in terms of Figure 4. During the anode offtime, the cations in solution diffuse into the deposition structure such that after each rest period, the region of the deposition structure is populated by cations. Here, the simulation cell resembles the one in Figure 4A, in which more cations are in a position to immediately deposit upon application of the overpotential. The on-time of the applied overpotential is thus used more efficiently than in the diffusionlimited process associated with continuous charging at high overpotentials.

Taken together, the results show that shorter and more widely spaced electrode pulse sequences lead to both improved deposition morphology (Figure 5A) and reduced anode ontimes (Figure 5C). Possible advantages of shorter anode ontimes are a reduction in undesirable side reactions, such as those associated with electrolyte decomposition, ${ }^{36-38}$ and prevention of thermal runaway. ${ }^{39}$ These benefits come with some increase in the total charging time (Figure 5B), but the penalty is generally small in comparison to $\gamma$; in particular, comparison of Figures $5 \mathrm{~A}, \mathrm{~B}$ indicates that for a given total charging time (or similarly, for a given average particle deposition rate), more widely spaced pulses lead to reduced dendrite formation.

Double Layers, Diffusion Timescales, and Pulse Charging. The previous section demonstrated that shorter pulse durations can lead to improved deposition structures and reduced anode on-times. However, in physical systems, $t_{\text {on }}$ is bounded from below due to the finite timescale associated with forming the electrical double layer (EDL). ${ }^{24}$ During each pulse, a double layer of charge forms on the surface of the anode, and a finite period of time is required for the EDL to achieve a sufficient potential to allow for cation reduction. This timescale typically limits the pulse frequency to a maximum value of approximately $10^{4} \mathrm{~Hz}$ (although this value can be increased slightly if high applied currents are used), ${ }^{7,40}$ such that waveforms for which $t_{\text {on }}>10^{-4} \mathrm{~s}$ must be used to achieve plating of metal atoms. The threshold value for $t_{\text {on }}$ is indicated by the vertical dashed line in Figure 6A. The blue curve in this figure replots the results for $\gamma=10$ from Figure 5A. It is clear that the threshold value for $t_{\text {on }}$ with these parameters allows for significantly denser deposition morphology than is obtained using a continuous applied overpotential with the same reaction probability, although it does not permit the full realization of the compact deposition morphologies that are achievable with shorter pulses.

Figure 6 also illustrates that changing the relative timescales associated with the material properties of the SEI and electrode will impact the viability of the pulse-charging strategy. For example, Figure 6A shows the effect of changing the cation diffusion coefficient $D$ while keeping all other parameters in the system unchanged (including $p=1$ ). With slower cation diffusion (green curve), the benefits of pulse charging with $t_{\text {on }}>$ $10^{-4} \mathrm{~s}$ are greater than those realizable with faster cation diffusion (red curve). Naturally, we recognize the practical difficulties associated with changing cation diffusivity in a physical system while leaving other timescales unchanged; nonetheless, these results emphasize that pulse charging provides a means of manipulating the competition between cation diffusion and reduction to mitigate the degree of dendrite formation.

Comparison of the points at which the curves in Figure 6A intersect the dashed line reveals that, for a given value of $t_{\mathrm{on}}$ increasing $D$ leads to an increase in the dendrite formation propensity. This result directly contrasts with the trend that is anticipated from the earlier analysis of electrodeposition under continuous charging. Indeed, in Figure $6 \mathrm{~B}$ we show that whereas pulse charging in the high-overpotential limit yields a positive correlation between $D$ and $\alpha$, no such correlation is obtained for continuous charging in the high-overpotential limit, and a negative correlation is obtained for continuous charging at finite overpotentials.

The mechanistic basis for the negative correlation between $D$ and $\alpha$ with continuous charging and $p<1$ (Figure 6B, blue) is easily understood in terms of the competition between diffusive and reactive timescales in the system; with increasing $D$, 

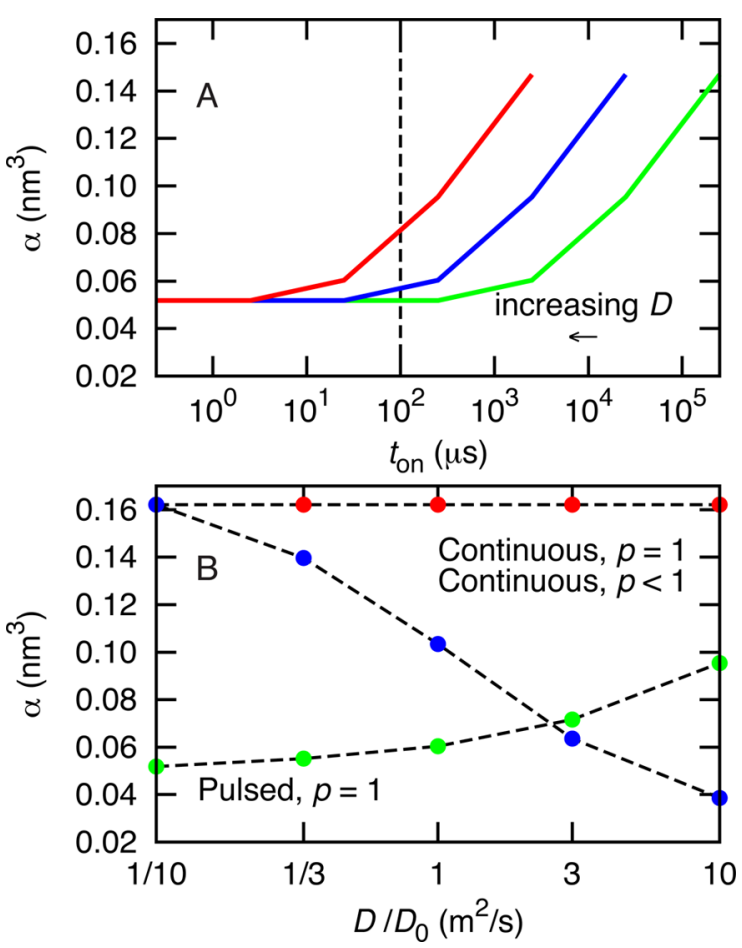

Figure 6. Relationship between pulse charging and cation diffusion. (A) Dendrite propensity coefficient $\alpha$ as a function of pulse duration $t_{\text {on }}$. The blue curve reproduces the results for the $\gamma=10$ curve in Figure 5A. The red and green curves correspond to the same simulation parameters, except with the cation diffusion coefficient, $D$, scaled by a factor of 10 and 0.1 , respectively. The vertical dashed line indicates the timescale for EDL formation. (B) Dendrite propensity coefficient $\alpha$ as a function of the cation diffusion coefficient, with $D_{0}=$ $1.40 \times 10^{-14} \mathrm{~m}^{2} / \mathrm{s}$. The green curve is obtained using pulse charging with $p=1, t_{\mathrm{on}}=253 \mu \mathrm{s}$, and $\gamma=10$. The red curve is obtained using continuous charging with $p=1$ (infinite $k_{\mathrm{ET}}$ ). The blue curve is obtained using continuous charging with $p<1\left(k_{\mathrm{ET}}=2.74 \times 10^{6} \mathrm{~s}^{-1}\right.$ for each point).

particles diffuse more deeply into the deposition structure on the timescale of a reactive event, leading to more compact structures and reduced dendrite formation. For continuous charging in the high-overpotential regime (Figure 6B, red), the timescale for reactive events is infinitely short in comparison to the timescale for diffusion (i.e., the system is diffusion limited), and changing the diffusion coefficient while staying in this regime has no impact on the resulting deposition structure.

The mechanistic basis for the positive correlation between $D$ and $\alpha$ with pulse charging (Figure 6B, green) is more interesting. As discussed previously, cation particles diffuse into the deposition structure between pulses; for any particular value of $D$, pulse charging thus leads to lower dendrite formation propensity than continuous charging (Figure 5A). In the high-overpotential limit, particles that have penetrated the deposition structure instantaneously undergo reaction when the electrode pulse is turned on; these "type A" reductive events lead to compact deposition structures. However, as the electrode pulse is left on, additional particles can diffuse from the surrounding bulk region and deposit at the outer reaches of the deposition structure; these "type B" reductive events lead to diffuse deposition structures. With increasing $D$, the cation particles undergo larger lengthscale motions within the fixed duration of the electrode pulse; this has little impact on the degree to which type A deposition occurs, but it enhances the degree to which type $\mathrm{B}$ deposition occurs. The net effect is increased dendrite propensity with increased cation diffusion, as seen in Figure 6B.

Finally, we note that trends observed in Figure $6 \mathrm{~B}$ have interesting consequences for the design of next-generation electrolyte materials. An important criterion in electrolyte design is the enhancement of ion diffusivity, which improves overall timescales for battery charging and discharging. The results presented here suggest that continued success in the development of conductive electrodes may somewhat diminish the degree of dendrite suppression to be gained from pulse charging. Conversely, these results also suggest that pulse charging offers greater dendrite suppression in electrolytes with lower diffusivity, which may help to compensate for the practical disadvantages of low diffusivity in some target applications.

\section{CONCLUSIONS}

An important thrust in the development of next-generation battery technologies is the emergence of new computational methods to bridge between molecular features of electrode chemistry and the long-timescale features of the charging/ discharging processes. CG approaches that incorporate microscopic parameters, such as cation diffusion coefficients and electrode/cation electron-transfer reaction rates, provide a useful step toward achieving these aims.

Here, we have introduced a CG simulation model to describe lithium metal electrodeposition and dendrite growth, and we have investigated the deposition morphology as a function of the magnitude and time dependence of the applied electrode overpotential. These simulations clearly demonstrate that dendrite formation propensity increases with electrode overpotential, although pulse charging can be used to significantly suppress dendrite formation at high overpotentials while also reducing the total required anode on-time. Analysis of the trajectories reveals that dendrite formation is fundamentally connected to the competition between the timescales for cation diffusion and reduction at the anode/SEI interface; the use of low overpotentials or short pulse durations shifts this competition in favor of cation diffusion and leads to lower dendrite formation propensity. Finally, we consider the interplay between cation diffusivity and deposition morphology, demonstrating that the time dependence of the electrode overpotential can lead to positive, negative, or zero correlation between cation diffusivity and dendrite formation propensity. This testable prediction from the CG model may provide a basis for future experimental studies of dendrite formation and electrolyte materials.

\section{AUTHOR INFORMATION}

\section{Corresponding Author}

*E-mail: tfm@caltech.edu.

\section{Notes}

The authors declare no competing financial interest.

\section{ACKNOWLEDGMENTS}

This work is supported in part by the U.S. Office of Naval Research (USONR) under Grant No. N00014-10-1-0884. Additionally, T.F.M. acknowledges support from a Camille and Henry Dreyfus Foundation New Faculty Award and an Alfred P. Sloan Foundation Research Fellowship. Computational resources were provided by the National Energy 
Research Scientific Computing Center, which is supported by the Office of Science of the U.S. Department of Energy under Contract No. DE-AC02-05CH11231, and by the National Science Foundation under Grant No. CHE-1040558. Finally, the authors thank Jason Goodpaster for helpful discussions at early stages of this work.

\section{REFERENCES}

(1) Tarascon, J. M.; Armand, M. Nature 2001, 414, 359-367.

(2) Kiehne, H. A. Battery Technology Handbook; CRC Press: Boca Raton, FL, 2003.

(3) Brissot, C.; Rosso, M.; Chazalviel, J. N.; Lascaud, S. J. Power Sources 1999, 81, 925-929.

(4) Aurbach, D.; Zinigrad, E.; Teller, H.; Dan, P. J. Electrochem. Soc. 2000, 147, 1274-1279.

(5) Monroe, C.; Newman, J. J. Electrochem. Soc. 2003, 150, A1377A1384.

(6) Crowther, O.; West, A. C. J. Electrochem. Soc. 2008, 155, A806A811.

(7) Chandrasekar, M. S.; Pushpavanam, M. Electrochim. Acta 2008, 53, 3313-3322.

(8) Adelkhani, H.; Ghaemi, M.; Jafari, S. M. J. Power Sources 2007, 163, 1091-1104.

(9) de Jongh, P. E.; Notten, P. H. L. Solid State Ionics 2002, 148, 259-268.

(10) Fukumoto, Y.; Kawashima, Y.; Hayashi, T. Surf. Coat. Technol. 1986, 27, 145-150.

(11) Kollia, C.; Loizos, Z.; Spyrellis, N. Surf. Coat. Technol. 1991, 45, 155-160.

(12) Nagy, G.; Sugimoto, Y.; Denuault, G. J. Electroanal. Chem. 1997, 433, 167-173.

(13) Magan, R. V.; Sureshkumar, R. Nanotechnology 2005, 16, S545S553.

(14) Magan, R. V.; Sureshkumar, R.; Lin, B. J. Phys. Chem. B 2003, 107, 10513-10520.

(15) Voss, R. F.; Tomkiewicz, M. J. Electrochem. Soc. 1985, 132, 371375.

(16) Ravi, R.; Raj, A. S.; Parthiban, T.; Radhakrishnan, G.; Kalidoss, R. Mater. Chem. Phys. 1993, 34, 290-294.

(17) Balbuena, P. B.; Wang, Y. Lithium-Ion Batteries: Solid-Electrolyte Interphase; Imperial College Press: London, 2004.

(18) Yamaki, J.; Tobishima, S.; Hayashi, K.; Saito, K.; Nemoto, Y.; Arakawa, M. J. Power Sources 1998, 74, 219-227.

(19) Funabiki, A.; Inaba, M.; Abe, T.; Ogumi, Z. J. Electrochem. Soc. 1999, 146, 2443-2448.

(20) Winter, M. Z. Phys. Chem. 2009, 223, 1395-1406.

(21) Fan, F. R. F.; Bard, A. J. Science 1997, 277, 1791-1793.

(22) Xiao, X. Y.; Bard, A. J. J. Am. Chem. Soc. 2007, 129, 9610-9612.

(23) Wessells, C. D.; Huggins, R. A.; Cui, Y. Nat. Commun. 2011, 2, 550.

(24) Bard, A. J.; Faulkner, L. R. Electrochemical Methods: Fundamentals and Applications, 2nd ed.; John Wiley \& Sons, Inc.: New York, 2001.

(25) Stone, G. M.; Mullin, S. A.; Teran, A. A.; Hallinan, D. T.; Minor, A. M.; Hexemer, A.; Balsara, N. P. J. Electrochem. Soc. 2012, 159, A222-A227.

(26) Allen, M. P.; Tildesley, D. J. Computer Simulation of Liquids; Oxford Science Publications: Oxford, U.K., 1989.

(27) Nimon, E. S.; Churikov, A. V. Electrochim. Acta 1996, 41, 14551464.

(28) Borodin, O.; Smith, G. D.; Fan, P. J. Phys. Chem. B 2006, 110, 22773-22779.

(29) Reed, S. K.; Lanning, O. J.; Madden, P. A. J. Chem. Phys. 2007, 126, 084704.

(30) Marcus, R. A.; Sutin, N. Biochim. Biophys. Acta 1985, 811, 265322.

(31) Monroe, C.; Newman, J. J. Electrochem. Soc. 2004, 151, A880A886.
(32) Kahanda, G. L. M. K. S.; Tomkiewicz, M. Phys. Rev. B 1988, 38, 957-959.

(33) Albertus, P.; Christensen, J.; Newman, J. J. Electrochem. Soc. 2008, 155, A48-A60.

(34) Tang, M.; Albertus, P.; Newman, J. J. Electrochem. Soc. 2009, 156, A390-A399.

(35) Sawada, Y.; Dougherty, A.; Gollub, J. P. Phys. Rev. Lett. 1986, 56, $1260-1263$

(36) Xing, L.; Borodin, O. Phys. Chem. Chem. Phys. 2012, 14, 1283812843.

(37) Xu, K. Chem. Rev. 2004, 104, 4303-4417.

(38) Yang, L.; Ravdel, B.; Lucht, B. L. Electrochem. Solid-State Lett 2010, 13, A95-A97.

(39) Maleki, H.; Deng, G. P.; Anani, A.; Howard, J. J. Electrochem. Soc. 1999, 146, 3224-3229.

(40) Roy, S.; Landolt, D. J. Appl. Electrochem. 1996, 27, 299-307. 